\title{
Cáncer renal incidental en pacientes menores de 40 años: hallazgos clínicos e histopatológicos
}

\author{
Gómez Pérez L*, Budía Alba A, Delgado Oliva FJ*, Ruiz Cerdá JL, Trassiera Villa M, Jiménez Cruz F.
}

Hospital Universitario La Fe. Valencia. *Hospital General Universitario de Elche. Alicante.

Actas Urol Esp 2007;31(3):244-249

\section{RESUMEN}

CÁNCER RENAL INCIDENTAL EN PACIENTES MENORES DE 40 AÑOS: HALLAZGOS CLÍNICOS E HISTOPATOLÓGICOS

Objetivo: Evaluar las características clínicas y patológicas del cáncer renal (CR) en nuestra serie de tumores, analizando su impacto en el grupo de edad de menores de 40 años.

Material y método: Se estudiaron 294 pacientes con CR. En 252 se realizó un estudio del ADN tumoral mediante citometría de flujo e histopatológico de las piezas quirúrgicas. Los pacientes fueron divididos en dos grupos en función de la edad, mayores y menores de 40 años. Las características clínicas e histopatológicas fueron comparadas entre ambos grupos.

Resultados: De los 294 pacientes, $26(8,94 \%)$ se incluyeron en el grupo de edad menor o igual a 40 años. No hallamos diferencias significativas entre los grupos al comparar estadio, tamaño tumoral o tratamiento realizado, aunque encontramos un mayor número de tumores indiferenciados en los pacientes de menor edad ( $\mathrm{p}=0,018)$. El análisis del $\mathrm{ADN}$ no evidenció diferencias en el porcentaje de aneuplodías al comparar ambos grupos. Tampoco encontramos diferencias al comparar la probabilidad de supervivencia actuarial cáncer específica en función de la edad.

Conclusiones: El comportamiento biológico del CR en pacientes menores de 40 años (jóvenes) no parece diferente al del grupo de mayor edad. Por ello pensamos que los esquemas de tratamiento y seguimiento no deben diferir en función de la variable edad.

Palabras clave: Cáncer renal. Edad. Análisis ADN.

\section{ABSTRACT}

INCIDENTAL RENAL CANCER IN YOUNG ADULTS: CLINICAL AND HISTOPATHOLOGICAL FINDINGS

Objective: to evaluate the clinical and pathological renal cancer (CR) characteristics in our series of tumours, analyzing its impact in the group of age less than 40 years.

Material and methods: We studied 294 patients with CR. The pathologic characteristics were analyzed and DNA ploidy pattern of the surgical pieces were done in 252 patients. The patients were divided in two groups based on age, greater or less to 40 years, well then clinical and pathologic characteristics were compared between.

Results: Of all patients, 26 of 294 patients $(8,94 \%)$ were included in the young age group (less to 40 years). We did not found differences between both groups comparing stage, tumoral volume, treatment realized or DNA ploidy pattern, but in nuclear grade with more aggressive tumours in young people ( $\mathrm{p}=0,0018$ ), without differences in recurrence-free survival or actuarial disease specific survival rate.

Conclusions: The findings in our study indicate that the natural history and outcome of the RC is similar in both older and younger patients. Therefore, in our opinion, the management of CR in young people should be established with independence of the age.

Keywords: Renal cancer. Age. DNA ploidy. 
$\mathrm{E}$ 1 cáncer renal supone más del 2-3\% de todas las neoplasias malignas de nuevo diagnóstico. El $80 \%$ de estos tumores se originan en el parénquima renal, siendo en su mayoría adenocarcinomas. El adenocarcinoma renal (CR) tiene una incidencia del 5\% aproximadamente y es responsable del 1-2\% de todas las muertes por cáncer $^{1}$. Dos tercios de estos tumores están localizados al diagnóstico y son potencialmente curables mediante tratamiento quirúrgico. Sin embargo el cáncer renal es el más letal de los cánceres urológicos, con una tasa de mortalidad que duplica la de otros tumores urológicos, incluyendo el de próstata o vejiga ${ }^{2}$. A pesar del tratamiento radical, el $40 \%$ desarrollarán metástasis y fallecerán ${ }^{3}$.

Los factores pronósticos que han demostrado su valor independiente de supervivencia y progresión en la mayoría de los estudios publicados en la literatura son el grado nuclear, el estadio al diagnóstico en la actual clasificación TNM $2002^{4,5}$, y la ploidía del ADN tumoral, aunque éste último todavía con un papel controvertido ${ }^{6}$. El tipo histológico también ha mostrado su valor pronóstico en algunos estudios tras su reciente reclasificación ${ }^{3}$. Sin embargo, la aplicación clínica de estos factores no consigue identificar con completa exactitud aquellos tumores que progresarán. Así, el 20-30\% de los tumores organoconfinados, a priori de buen pronóstico, desarrollarán metástasis y fallecerán por dicha causa ${ }^{7}$. Por ello son necesarios más estudios que aporten más información y permitan identificar con mayor exactitud ese grupo de pacientes candidatos a un seguimiento más estricto o a terapias adyuvantes $^{8,9}$. Recientemente, en un intento de mejorar la predicción pronóstica han surgido nuevos sistemas integrados de estadificación, combinando el TNM con otras variables clínicas y patológicas, que permiten estratificar en categorías pronósticas, calculando mediante herramientas estadísticas la probabilidad de supervivencia para cada paciente ${ }^{10}$.

Otro factor analizado en la literatura ha sido la influencia de la edad en las características clínicas y tumorales del CR. Así recientes trabajos han evaluado la variable edad en el CR e incluso han caracterizado los tipos histológicos más frecuentes ${ }^{11}$, riesgo de recurrencia ${ }^{3}$ e implicación pronóstica $^{12}$ en función del grupo de edad al que pertenecen. Sin embargo los resultados publicados son controvertidos.

El objetivo de este estudio ha sido evaluar la influencia de la variable edad (pacientes jóvenes menores de 40 años) en las características clínico-patológicas del CR en nuestra serie de tumores.

\section{MATERIAL Y MÉTODO}

Se han estudiado de forma retrospectiva a 294 pacientes diagnosticados de CR y tratados quirúrgicamente en nuestro Servicio entre los años 1969 a 2001.

En 252 de los pacientes incluidos, se realizó un estudio del contenido de ADN tumoral mediante citometría de flujo. Dicho estudio fue realizado en el laboratorio de citometría de flujo del servicio de Hematología de nuestro hospital. El análisis nuclear del ADN se realizó sobre células obtenidas de muestras en fresco de la pieza quirúrgica en los pacientes más recientes (19962001) y sobre muestras desparafinadas en los pacientes anteriores a 1996. Las muestras frescas fueron disgregadas mecánicamente, filtradas, lavadas y centrifugadas. A partir de $100 \mu \mathrm{L}$ de dicha muestra, se añadía el reactivo comercial Coulter DNA-Prep Reagent Kit (Coulter Corporation, Miami, USA) previo a la lectura por el citómetro de flujo Cytoron Absolute (Orthodiagnosis Systems). Las muestras parafinadas se trataron siguiendo la técnica de Hedley ${ }^{13}$. Las muestras tumorales fueron consideradas diploides cuando el índice de $\mathrm{ADN}$ estaba entre 0,9 y 1,1 (cociente entre el canal del pico $G_{0} / G_{1}$ de la muestra y el canal del pico $G_{0} / G_{1}$ de las células control diploides normales). Además las muestras fueron consideradas diploides cuando sólo un pico era detectado en el análisis y aneuploides cuando se hallaban 2 o más picos. La mezcla de una muestra control con la del paciente nos permitía confirmar si se trataba de una hipo o hiperploidía.

$\mathrm{El}$ estudio anátomo-patológico fue realizado por el Servicio de anatomía patológica de nuestro hospital, tras la extracción de la pieza quirúrgica. El estudio de extensión anatómica tumoral se reclasificó siguiendo los criterios de la clasificación de consenso TNM 2002 de la Internacional Union Against Cancer. Los tumores se clasifica- 
ron como organoconfinados (estadio I-II) y no organoconfinados (estadio III-IV) para su comparación. El grado nuclear fue evaluado con el sistema de gradación descrito por Furhman ${ }^{14}$. Para su comparación los tumores se agruparon como de bajo grado (grado I-II) y de alto grado (grado III-IV).

El seguimiento de los pacientes se realizó de acuerdo a los protocolos oncológicos de nuestro hospital, con controles cuatrimestrales el primer año, semestrales hasta el $5^{\circ}$ año y anuales a partir del $5^{\circ}$ año. La progresión clínica fue definida como recurrencia local, regional y/o presencia de enfermedad a distancia.

Para evaluar la variable edad en el CR, los pacientes fueron divididos en dos grupos: menores o iguales a 40 años (grupo I) y mayores de 40 años (grupo II). Las características clínicas y tumorales fueron comparadas entre ambos grupos.

El análisis estadístico se realizó con programa estadístico SPSS versión 12.0 (SPSS, Inc., Chicago, Illinois). El valor de p considerado significativo fue menor de 0,05. Las variables a estudio fueron categorizadas siguiendo criterios clinicos o de tamaño muestral. Para las comparaciones de las características clínicas e histopatológicas entre los grupos de edad definidos, se utilizaron las pruebas de Chi cuadrado y el test de Fischer cuando algunas de las frecuencias esperadas era menor de 5. La supervivencia actuarial cáncer específica fue calculada con las curvas de Kaplan Meyer y el test de log- rank para la comparación de grupos.

\section{RESULTADOS}

De los 294 pacientes, 24 (8,16\%) pertenecían al grupo I (26 a 40 años, con mediana de edad de 36 años) y $270(91,8 \%)$ se incluyeron en el grupo II (de 41 a 83 años, con mediana de edad 61 años) en el momento del diagnóstico. La mediana de seguimiento fue de 53,5 (2-204) meses. El estudio descriptivo de la muestra se describe en la Tabla 1 .

Cuando se evaluó el estadio patológico, categorizado en organoconfinados y no organoconfinados, se observó que en el grupo I los tumores estaban organoconfinados al diagnóstico en 13 pacientes $(54,16 \%)$, frente a los 128 pacientes
Tabla 1

Características clínico tumorales en función del grupo de edad.

\begin{tabular}{|c|c|c|}
\hline $\begin{array}{l}\text { Caracteristicas } \\
\text { Clinico-Tumorales }\end{array}$ & $\begin{array}{c}\text { Grupo I } \\
(\leq 40 \text { años })\end{array}$ & $\begin{array}{c}\text { Grupo II } \\
\text { (> } 40 \text { años })\end{array}$ \\
\hline Pacientes n (\%) & $24(8,16)$ & $270(91,8)$ \\
\hline $\begin{array}{l}\text { Edad } \\
\text { (mediana-rango) }\end{array}$ & 36 (26-40) años & 61 (41-83) años \\
\hline CR convencional & $16(66,66 \%)$ & $164(60,74 \%)$ \\
\hline CR papilar & $4(16,66 \%)$ & $48(17,77 \%)$ \\
\hline CR cromofobo & $4 \quad(16,66 \%)$ & $58(21,48 \%)$ \\
\hline CR indiferenciado & $0 \quad(0 \%)$ & $0(0 \%)$ \\
\hline Grado I-II & $12(50 \%)$ & $111(37,3 \%)$ \\
\hline Grado III-IV & $12(50 \%)$ & $169(62,59 \%)$ \\
\hline $\begin{array}{l}\text { Tamaño tumoral } \\
\text { (mediana-rango) }\end{array}$ & $7(2-20) \mathrm{cm}$ & $7(2-28) \mathrm{cm}$ \\
\hline Tumores diploides & $14(58,33 \%)$ & $154(57,03 \%)$ \\
\hline Tumores aneuploides & $10(41,66 \%)$ & $116(42,96 \%)$ \\
\hline
\end{tabular}

$(47,4 \%)$ del grupo II $(\mathrm{p}=0,532)$. El estadio patológico más frecuente fue el III en ambos grupos (Tabla 1). No se encontraron diferencias de significación estadística en el porcentaje de pacientes con adenopatías presentes al diagnóstico (25\% en el grupo I y 20,8\% en el grupo II, $\mathrm{p}=0,611$ ), ni con metástasis (4,2\% en el grupo I y $6,7 \%$ en el grupo II, $\mathrm{p}=0,630$ ).

La comparación del grado nuclear de Furhman en función del grupo de edad, mostró mayor porcentaje de tumores indiferenciados en el grupo I (50\%) frente al grupo II (26,6\%) con diferencias estadísticamente significativas $(p=0,018)$. El tipo histológico más frecuente fue el CR convencional en ambos grupos.

El análisis de la ploidía del $\mathrm{ADN}$ no mostró diferencias significativas al comparar ambos grupos $(p=0,995)$. Cuando estudiamos el porcentaje de aneuplodías en función de si el tumor era o no órgano confinado, observamos mayor índice de aneuploidías en tumores no órgano confinados, sin encontrar diferencias significativas al comparar el porcentaje de aneuploidías en función de la edad (Tabla 2).

La mediana del tamaño tumoral en el grupo I fue de 7 (rango 2-20) al igual que el grupo II, que también fue 7 (rango 2-28), sin diferencias significativas al comparar ambos grupos en función del tamaño tumoral $(\mathrm{p}=0,982)$. 
Tabla 2

Distribución de pacientes según porcentaje de aneuploidías, organoconfinamiento y grupo de edad.

\begin{tabular}{lcc}
\hline $\begin{array}{l}\text { Grupo } \\
\text { de edad }\end{array}$ & $\begin{array}{c}\text { Órgano } \\
\text { confinados } \\
\text { (\% aneuploidias) }\end{array}$ & $\begin{array}{c}\text { No órgano } \\
\text { (\% aneuploidias })\end{array}$ \\
\hline Edad $\leq 40$ años & $4(36,4)$ & $5(50)$ \\
Edad $>40$ años & $27(26,5)$ & $67(56,8)$ \\
Valor $\mathrm{p}$ & 0,485 & 0,656 \\
\hline
\end{tabular}

En el grupo II, 262 pacientes (97,03\%) fueron tratados mediante nefrectomía radical y 8 $(2,96 \%)$ con cirugía conservadora. Respecto grupo I, en 22 pacientes $(91,66 \%)$ se realizó cirugía radical y en el resto cirugía conservadora (tumorectomía o nefrectomía parcial). Al establecer la comparación entre grupos de edad, observamos diferencias significativas $(p=0,001)$ por el mayor porcentaje de cirugías conservadoras en el grupo I.

Durante el seguimiento a largo plazo, el tumor progresó en 89 pacientes $(36,29 \%)$ del grupo II frente a $7(29,2 \%)$ del grupo I $(p=0,882)$. El mayor porcentaje de progresión tumoral se observó en los tumores no organoconfinados, siendo mayor en el grupo II respecto al grupo I (49,8\% frente al $36,4 \%$ ), aunque estas diferencias no alcanzaban la significación estadística $(\mathrm{p}=0,682)$.

La supervivencia actuarial cáncer específica (Fig. 1), no mostró diferencias estadísticamente significativas entre ambos grupos de pacientes

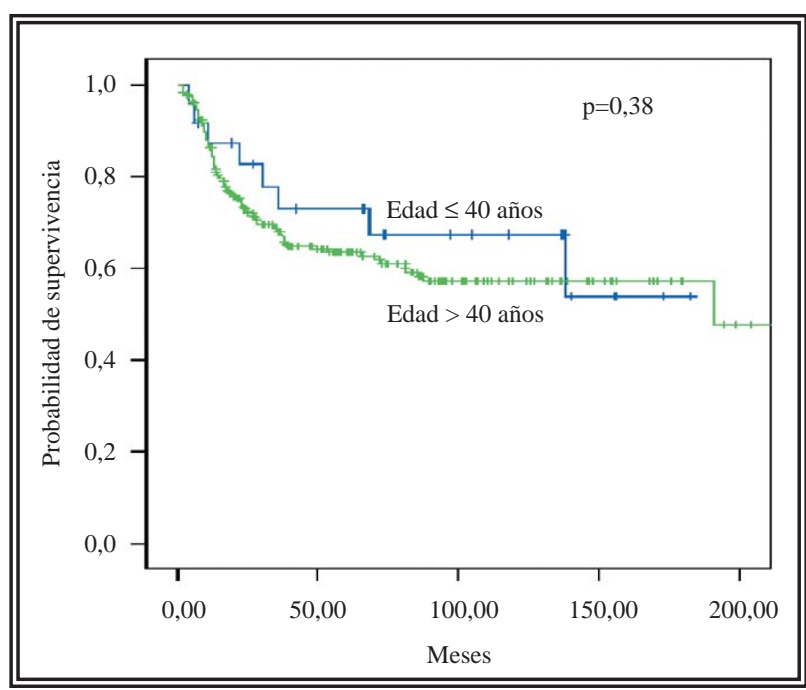

FIGURA 1. Supervivencia actuarial cáncer específica según grupo de edad $(\mathrm{p}=0,38)$, con probabilidad de supervivencia a 5 años de $67,6 \%$ y $61,5 \%$ para menores y mayores de 40 años respectivamente. La supervivencia actuarial cáncer específica en tumores organoconfinados y no organoconfinados, no mostró diferencias significativas entre ambos grupos de edad (Figs. 2 y 3).

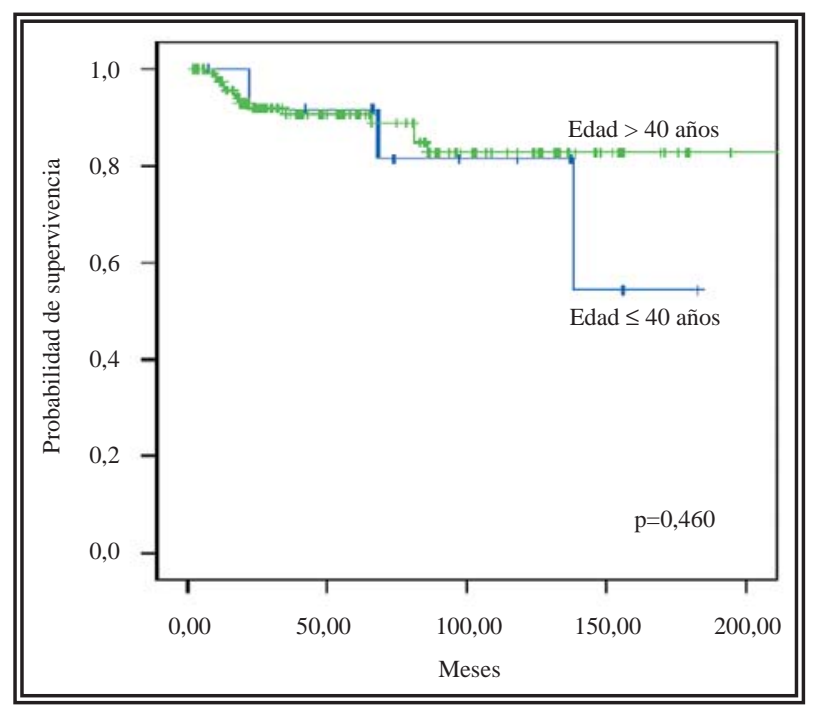

FIGURA 2. Supervivencia actuarial cáncer específica según grupo de edad en tumores organoconfinados.

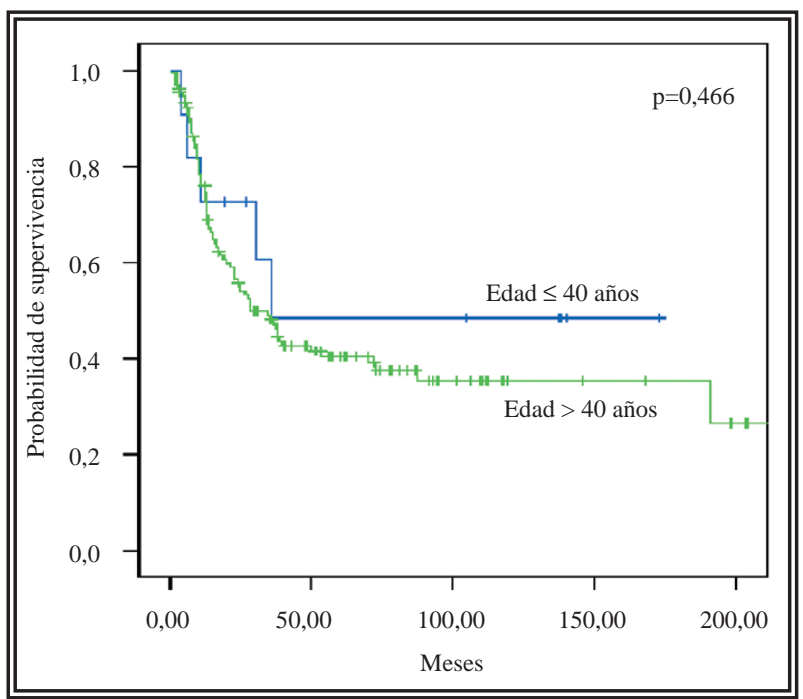

FIGURA 3. Supervivencia actuarial cáncer específica según grupo de edad en tumores no organoconfinados.

\section{DISCUSIÓN}

Comprender el comportamiento biológico del CR en adultos jóvenes tiene gran importancia clínica, con implicaciones en el tratamiento y seguimiento ${ }^{1}$. 
Sin embargo la baja incidencia de esta patologia en menores de 40 años dificulta conocer la evolución natural en este grupo de edad. En dos grandes series de los años 80, Kantor ${ }^{15}$ encontró que de 3766 pacientes con CR en $127(3,4 \%)$ se presentaba antes de los 40 años. Lieber ${ }^{16}$ refiere una incidencia similar $(3,5 \%)$ en su serie de 3768 pacientes. Sin embargo, al revisar la mayoría de las series actuales ${ }^{17,18}$, apreciamos un incremento paulatino en la incidencia del CR, que llega hasta el 8,7\% en nuestra muestra. Este aumento puede ser debido a una mayor probabilidad del diagnóstico incidental, por la utilización rutinaria de técnicas radiológicas como ecografia, TAC y RNM para el estudio de otras patologias y a la mayor presencia de potenciales factores etiológicos como el hábito tabáquico.

Algunos autores como Sánchez-Ortíz y Schiff ${ }^{19,20}$, encontraron diferencias en el estadio del paciente al diagnóstico en función de la edad, con un mayor porcentaje tanto de afectación linfática como de presencia de metástasis en pacientes jóvenes. Este hallazgo sugería un comportamiento biológico diferente del $\mathrm{CR}$ en este grupo de edad. En nuestro estudio no hemos apreciado estas diferencias en el comportamiento biológico del tumor entre los dos grupos de edad. Una posible explicación estaría en la selección de pacientes. En nuestra serie, los dos grupos de pacientes son homogéneos y comparables al no existir diferencias en la distribución por estadios. Sin embargo la serie de Sánchez-Ortiz ${ }^{19}$ incluye un elevado porcentaje de pacientes con enfermedad localmente avanzada o con metástasis, remitidos a su centro para ensayo de nuevas terapias, lo que podria haber sesgado los resultados. Por otra parte, la serie de Schiff ${ }^{20}$ está basada en un registro histórico de los años 80, no comparable con series actuales en las que el porcentaje de tumores incidentales es mayor y la capacidad de detección diagnóstica tumoral ha aumentado.

Respecto al contenido celular de ADN de la célula tumoral, no encontramos que los tumores en el grupo de edad más joven mostraran un comportamiento más agresivo en el análisis de la ploidía del ADN. La aneuploidía tumoral mostró asociación con el estadio pero no con la edad, como ya fue descritopor nuestro grupo ${ }^{21}$. Similares hallazgos se obtuvieron en el análisis de la serie de la Clínica Mayo ${ }^{22}$ en un estudio de variables pronósticas del CR en pacientes jóvenes.
Sin embargo, sí hemos encontrado asociación entre la edad y el grado de diferenciación tumoral. Se observó una mayor proporción de tumores indiferenciados en pacientes jóvenes. Esta asociación ha sido descrita previamente por Renshaw $^{11}$, aunque con pacientes en rango de edad entre 6 y 29 años, lo que explicaría que también encontremos diferencias en el tipo histológico más frecuente (el papilar), respecto a nuestros resultados. Al igual que en nuestra serie, otros autore ${ }^{12,16,23}$ no encontraron estas diferencias en el tipo histológico según la edad, siendo el convencional el más frecuente.

Cuando analizamos en la literatura la progresión tumoral o probabilidad de supervivencia cáncer específica en función de la edad, en general se aprecia una mayor supervivencia de los pacientes jóvenes con $\mathrm{CR}^{24,25}$, pero sólo demostrada en las series de Yusim ${ }^{26}$ y Sánchez-Ortíz ${ }^{19}$. Nosotros encontramos resultados similares, pero con una probabilidad de supervivencia algo inferior, probablemente porque el $45 \%$ de los tumores en este grupo de edad tienen un estadio $\mathrm{pT}_{3 \mathrm{a}}$ o superior en nuestra serie.

En conclusión y según nuestros resultados, el comportamiento biológico del $\mathrm{CR}$ en pacientes menores de 40 años (jóvenes) no parece diferente al del grupo de mayor edad. Por ello pensamos que los esquemas de tratamiento y seguimiento no deben diferir en función de la variable edad.

\section{REFERENCIAS}

1. Goetzl MA, Desai M, Mansukhani M, Goluboff ET, Katz AE, Sawczuk IS, et al. Natural history and clinical outcome of sporadic renal cortical tumors. Urology. 2004;63(1):41-45.

2. Lam J, Leppert J, Belldegrun A, Figlin RA. Adjuvant therapy of renal cell carcinoma: patient selection and therapeutic options. BJU. 2005;96(4):483-488.

3. Griffiths DF, Verghese A, Golash A, Kynaston HG, Matthews PN, Hart AJ, et al. Contribution of grade, vascular invasion and age to outcome in clinically localized renal cell carcinoma. BJU Int. 2002;90(1):26-31.

4. Ficarra V, Prayer-Galetti T, Novella G, Bratti E, Maffei N, Dal Bianco M, et al. Incidental detection beyond pathological factors as prognostic predictor of renal cell carcinoma. Eur Urol. 2003;43(6):663-669.

5. Mejean A, Hopirtean V, Bazin JP, Larousserie F, Benoit H, Chretien Y, et al. Prognostic factors for the survival of patients with papillary renal cell carcinoma: meaning of histological typing and multifocality. J Urol. 2003;170(3):764-767.

6. Sanchez Zalabardo D, Arocena Garcia-Tapia J, Regojo Balboa JM, Fernandez Montero JM, Lopez Ferrandis J, Rosell Costa D, et al. Factores pronóstico en Carcinoma Renal Pt3. Actas Urol Esp. 2003;27(1):26-32. 
7. Di Silverio F, Casale P, Colella D, Andrea L, Seccareccia F, Sciarra A, et al. Independent value of tumor size and DNA ploidy for the prediction of disease progression in patients with organ-confined renal cell carcinoma. Cancer. 2000; 88(4):835-843.

8. Delahunt B. Histopathologic prognostic indicators for renal cell carcinoma. Sem Diag Pathol. 1998;15(1):68-76.

9. Gelb AB. Renal cell carcinoma: current prognostic factors. Union Internationale Contre le Cancer (UICC) and the American Joint Committee on Cancer (AJCC). Cancer. 1997;80(5):981-986.

10. Lam JS, Shvarts O, Leppert JT, Figlin RA, Belldegrun AS. Renal cell carcinoma 2005: new frontiers in staging, prognostication, and targeted molecular therapy. J Urol. 2005; 173(6): 1853-1862.

11. Renshaw AA, Granter SR, Fletcher JA, Kozakewich HP, Corless CL, Perez-Atayde AR. Renal cell carcinomas in children and young adults: increased incidence of papillary architecture and unique subtypes. Am J Surg Pathol. 1999;23(7):795-802.

12. Boykin WH, Bright KE, Zeidman EJ, Thompson IM. Renal tumors in young adults. Urology. 1992;40(6):503-505.

13. Hedley D, Friedlander M, Tylor IW. Application of DNA flow cytometry to paraffin embedded archival material for the study of aneuploidy and its clinical significance. Cytometry. 1985;6(4):327-333.

14. Fuhrman S, Lasky L, Limas C. Prognostic significance of morphologic parameters in renal cell carcinoma. Am J Surg Pathol. 1982;6(7):651-661.

15. Kantor AL, Meigs JW, Heston JF, Flannery JT. Epidemiology of renal cell carcinoma in Connecticut, 1935-1973. J Natl Cancer Inst. 1976;57(3):495-500.

16. Lieber M, Tomera F, Taylor W, Farrow GM. Renal adenocarcinoma in young adults: survival and variables affecting prognosis. J Urol. 1981;125(2):164-169.

17. Rodriguez A, Patard JJ, Lobel B. Renal cell carcinoma in young adults: incidence, disease outcome and review of the literature. Arch Esp Urol. 2002;55(8):969-975.

18. Siemer S, Hack M, Lehmann J, Becker F, Stockle M. Outcome of renal tumors in young adults. J Urol. 2006; 175(4): 1240-1244
19. Sanchez-Ortiz RF, Rosser CJ, Madsen LT, Swanson DA, Wood CG. Young age is an independent prognostic factor for survival of sporadic renal cell carcinoma. J Urol. 2004; 171(6 Pt 1):2160-2165.

20. Schiff M, Herter G, Lytton B. Renal adenocarcinoma in young adults. Urology. 1985;25(4):357-359.

21. Ruiz Cerdá JL, Hernández Martí M, Sempere Talens A, Martíinez Jabaloyas J, Rodrigo Aliada M, Monros Lliso JL et al. Estudio del contenido en ADN y de su asociación con el estadio, tamaño tumoral, arquitectura, tipo celular y grado nuclear en el adenocarcinoma renal. Actas Urol Esp. 1997;21(1):6-14.

22. Rainwater LM, Zincke H, Farrow GM, Gonchoroff NJ. Renal cell carcinoma in young and old patients. Comparison of prognostic pathologic variables (cell type, tumor grade and stage and DNA ploidy pattern) and their impact on disease outcome. Urology. 1991;38(1):1-5.

23. Abou El Fettouh HI, Cherullo EE, El-Jack M, Al Maslamani Y, Novick AC. Sporadic renal cell carcinoma in young adults: presentation, treatment and outcome. Urology. 2002; 60(5):806-811.

24. Gillett MD, Cheville JC, Karnes RJ, Lohse CM, Kwon ED, Leibovich BC, et al. Comparison of presentation and outcome for patients 18 to 40 and 60 to 70 years old with solid masses. J Urol. 2005;173(6): 1893-1896.

25. Eggener SE, Rubenstein JN, Smith ND, Nadler RB, Kontak $\mathrm{J}$, Flanigan RC, et al. Renal tumors in young adults. J Urol. 2004;171(1):106-110.

26. Yusim I, Mermershtain W, Neulander E, Eidelberg I, Gusakova I, Kaneti J, et al. Influence of age on the prognosis of patients with renal cell carcinoma (RCC). Onkologie 2002;25(6):548-552.

Correspondencia autor: Dr. L. Gómez Pérez

Servicio de Urología. Hospital General Universitario de Elche Camí de l'Almassera, 11. 03203 Elche (Alicante)

Tel.: 966679000

E-mail autor: luisgope@hotmail.com

Información artículo: Original - Cáncer renal

Trabajo recibido: agosto 2006

Trabajo aceptado: noviembre 2006 